\title{
On the possibility of using an electromagnetic ionosphere in global MHD simulations
}

\author{
P. Janhunen \\ Finnish Meteorological Institute, Geophysical Research, P.O. Box 503, FIN-00101 Helsinki, Finland
}

Received: 14 January 1997 / Revised: 22 September 1997 / Accepted: 3 October 1997

\begin{abstract}
Global magnetohydrodynamic (MHD) simulations of the Earth's magnetosphere must be coupled with a dynamical ionospheric module in order to give realistic results. The usual approach is to compute the field-aligned current (FAC) from the magnetospheric MHD variables at the ionospheric boundary. The ionospheric potential is solved from an elliptic equation using the FAC as a source term. The plasma velocity at the boundary is the $\mathbf{E} \times \mathbf{B}$ velocity associated with the ionospheric potential. Contemporary global MHD simulations which include a serious ionospheric model use this method, which we call the electrostatic approach in this paper. We study the possibility of reversing the flow of information through the ionosphere: the magnetosphere gives the electric field to the ionosphere. The field is not necessarily electrostatic, thus we will call this scheme electromagnetic. The electric field determines the horizontal ionospheric current. The divergence of the horizontal current gives the FAC, which is used as a boundary condition for MHD equations. We derive the necessary formulas and discuss the validity of the approximations necessarily involved. It is concluded that the electromagnetic ionosphere-magnetosphere coupling scheme is a serious candidate for future global MHD simulators, although a few problem areas still remain. At minimum, it should be investigated further to discover whether there are any differences in the simulation using the electrostatic or the electromagnetic ionospheric coupling.
\end{abstract}

Key words. Ionosphere - Ionosphere-magnetosphere interaction - Magnetospheric physics - Magnetosphereionosphere interaction. Space plasma physics .

Numerical simulation studies

\section{Introduction}

Global magnetohydrodynamic (MHD) simulations of the Earth's magnetosphere, and possibly other celestial objects, are becoming powerful tools for many kinds of quantitative studies (e.g., Raeder et al., 1995, Fedder et al., 1995, Tanaka, 1995, Janhunen et al., 1996, Janhunen, 1996). To yield realistic results, however, it is necessary to couple these models to a detailed ionospheric model, which is a nontrivial task even at the conceptual level, as will become apparent.

An electrostatic ionosphere coupled to global MHD simulations was recently discussed in detail by Goodman (1995) [see also Amm (1996) and Goodman (1996)]. Besides, the electrostatic ionosphere has been implemented by at least some of the groups working on global MHD studies (Raeder et al., 1995, Fedder et al., 1995, Janhunen, 1996). In the present paper our focus is not the ionospheric elliptic equation but rather the magnetosphere-ionosphere interface boundary.

The structure of the paper is as follows. We first present the electrostatic ionospheric model. We are most familiar with our own simulation code (GUMICS-3) (Janhunen, 1996), and consequently our presentation of the electrostatic model follows closely the implementation found in GUMICS-3. We then propose the new electromagnetic model (that is, allow a nonzero curl for the ionospheric electric field), deriving the necessary field-aligned current (FAC) mapping formulas. We conclude the paper by discussing the physical meaning of the two models and their equivalence.

\section{The electrostatic ionosphere}

When coupling global MHD simulations with ionospheric models, it is customary to introduce an artificial boundary $(\mathrm{AB})$ above the ionosphere. In our model the $\mathrm{AB}$ is located at $3.7 R_{E}$. This is done to avoid solving the
Correspondence to: Pekka Janhunen
Fax: + 35891929 4603; e-mail: pekka.janhunen@fmi.fi

(Also at Swedish Institute of Space Physics, Uppsala, Sweden) 
MHD equations in the near-Earth region, where the very high Alfvén speed would force us to use a very small time-step to obey the Courant condition (the Courant condition requires that the time-step must be smaller than the fast magnetosonic wave travel time through the grid cell, otherwise numerical instability will result). The region between the ionosphere and the $\mathrm{AB}$ is assumed force-free, which means that all current is fieldaligned and the magnetic field is approximated by the dipole field (in principle, quadrupole and higher terms representing the internal field could also be included). In GUMICS-3 we use adaptive subcycling in time, so that while the time-step is small for near-Earth grid cells, it remains larger for other cells. This optimization minimizes the computational burden resulting from the high near-Earth Alfvén speed.

The location of the $\mathrm{AB}$ is a trade-off between resolution and geometric distortions. If the $\mathrm{AB}$ is located at low altitude, $2 R_{E}$, say, then the distortions resulting from the fact that the magnetic field is not exactly force-free and the variation field is not necessarily very much smaller than the dipole field, are reduced. However, the simulation code is slowed down a lot. It is also more difficult to achieve high spatial resolution at the $\mathrm{AB}$ and in the ionosphere (ionospheric and $\mathrm{AB}$ resolution are related by flux-tube mapping): to maintain the same mapped-down ionospheric resolution, the spacing between grid points at the AB must be lowered further, which increases the computational load even further. We have preferred to set the $\mathrm{AB}$ at a relatively high $\left(3.7 R_{E}\right)$ altitude and to accept that some distortions might occur during strong FAC events. The saved CPU time has been used to increased spatial resolution in the whole magnetosphere. In this way the simulation as a whole has a relatively high resolution, especially in the near-Earth region, which means that more physical phenomena can be addressed. We believe that the distortions due to the $\mathrm{AB}$, being of geometric nature, do not affect the results qualitatively.

A possibility that has not yet been studied is whether an implicit MHD code could be used. In case of implicit codes we would not have to obey the Courant condition and the $\mathrm{AB}$ could be placed at a low altitude, possibly even at the ionosphere. However, the propagation of Alfvén waves would not be accurately resolved in the near-Earth region in this case.

The computational magnetosphere-ionosphere coupling cycle starts from the $\mathrm{AB}$, where the $\mathrm{FAC}$ is computed from the MHD variables: $j_{\|}=\hat{\mathbf{B}} \cdot \nabla \times \mathbf{B} / \mu_{0}$, where $\hat{\mathbf{B}}$ denotes the unit vector in the direction of the magnetic field. Within the force-free region the FAC (which is a scalar) can be mapped along the magnetic field by multiplying it by the magnetic field ratio, because the total current is always divergence-free (the Debye length is always much smaller than the scales of interest in global MHD studies) and within the forcefree region, all current is by definition FAC. Being a low-beta plasma, the near-Earth region is force-free because any perpendicular component of the current density $\mathbf{j}$ would introduce a $\mathbf{j} \times \mathbf{B}$ force too large to be canceled by the pressure gradient or other terms in the momentum equation (Janhunen and Koskinen, 1997). Thus we can easily obtain the FAC at the ionospheric plane $j_{\|}^{(i)}$.

The next step is to solve the ionospheric potential $\Phi_{i}$ from the elliptic equation (for example, Amm, 1996)

$-\nabla \cdot\left(\Sigma \cdot \nabla \Phi_{i}\right)=j_{\|}^{(i)} \sin I$

where $\Sigma$ is the horizontal height-integrated conductivity tensor and $I$ is the inclination angle of the magnetic field. In GUMICS-3 the conductivities depend on space and time according to solar UV radiation and electron precipitation which is approximately computed from the self-consistent MHD variables (Janhunen, 1996). The FAC $j_{\|}^{(i)}$ is measured positive downward. The LHS of Eq. (1) is the divergence of the horizontal ionospheric current $\left(\mathbf{J}=\Sigma \cdot \mathbf{E}=-\Sigma \cdot \nabla \Phi_{i}\right.$ is the horizontal current). If the neutral atmosphere is moving, the term $\mathbf{v}_{n} \times \mathbf{B}\left(\mathbf{v}_{n}\right.$ is the neutral velocity) must be added to $\mathbf{E}$; this is also one way to take into account Earth's rotation. The term containing $\mathbf{v}_{n}$ is transferred to the RHS of the equation because it is a source term.

The solution of Eq. (1) requires an elliptic solver. In GUMICS-3 we use the biconjugate gradient method (Press et al., 1992). If polar coordinates are used, the poles may cause some problems because the coordinates are singular there. Nevertheless, in GUMICS-3 we are currently using polar coordinates and solving for the northern and southern hemispheric potential separately to reduce the size of the linear system. We put $\Phi_{i}$ to zero at the pole (homogeneous Dirichlet condition) and use the homogeneous Neumann boundary condition (zero latitudinal derivative of the potential) at the equatorward boundary. The equatorward boundary resides at about $50^{\circ}$ magnetic latitude; the regions equatorward from this circle do not map to the AB surface. In this way the additive constants are effectively fixed separately on both the hemispheres, but this causes no problems because the constants are differentiated away later (as we will show). In this case we found that using the "conservative" differencing [i.e., differencing Eq. (1) as such without expanding the differentiations analytically first, see also Oppenheim (1995)] reduces the problems caused by the coordinate singularity at the north/south pole such that only a slight kink in the potential contours is sometimes seen at the pole, which is only an aesthetic problem.

When the ionospheric potential is known, it is mapped through the force-free region to the $\mathrm{AB}$ (artificial boundary), taking into account the parallel potential drop contribution also. If there is no parallel potential drop, the potential is mapped by simply copying it along the dipole field line. We will assume that this is the case for the moment and return to the parallel potential drop issue later. Thus we obtain the potential at the $\mathrm{AB}$, which enables us to compute the corresponding electric field $\mathbf{E}$ by differentiation. It is at this stage that the additive constants disappear and no problems result from the separate treatment of the two hemispheres. Having the electric field at the $\mathrm{AB}$ we compute the corresponding $\mathbf{E} \times \mathbf{B}$ velocity 
$\mathbf{v}=\mathbf{E} \times \mathbf{B} / B^{2}$, which is used as a boundary condition for the MHD equations. This fixes the perpendicular velocity at the AB. To fix the magnetic field-parallel velocity, three approaches have been tested in GUMICS-3: (1) let the parallel velocity be zero, (2) use a homogeneous Neumann boundary condition for the parallel velocity, i.e., copy the parallel velocity from the nearest interior grid cell, (3) use such a value for the parallel velocity that the component normal to the AB surface is zero. Because the magnetic field is not exactly radial at $\mathrm{AB},(3)$ is not the same as (1), although in polar regions the difference is not large. Approach (1) works reasonably well in most cases, but during long periods of positive IMF $B_{z}$, unrealistically large plasma density accumulation may occur in the dayside cusp region. Approach (2) does not seem to give good results. Approach (3) is currently being tested. Overall, the question of plasma flow across the $\mathrm{AB}$ is still an obscure one which would deserve further study. However, relatively short phenomena such as substorms are likely not to be very sensitive on plasma transport across the AB.

The ionosphere-magnetosphere interaction cycle in the electrostatic approach is now complete. We started from magnetospheric FAC and arrived at plasma velocity at the AB surface. In GUMICS-3 the coupling cycle is computed every $4 \mathrm{~s}$. With this setup, the program spends $80-90 \%$ of the time in the magnetosphere and $10-20 \%$ of the time in the ionosphere. Within the ionosphere, the iterative solution of the elliptic equation dominates the CPU time.

The remaining issue is how to include the parallel potential drop. It can be taken into account by adding its contribution to the ionospheric potential $\Phi_{i}$. If only the mirror force on electrons is taken into account, the potential at the AB surface is the mapped-up version of $\Phi_{m} \equiv \Phi_{i}+\Delta \Phi$, where $\Delta \Phi$ is equal to

$\Delta \Phi=\min \left(0, \frac{j_{\|}^{(i)}}{K}+\frac{k_{B} T}{e}\right)$

(Janhunen, 1996), where positive current is downward and $\Delta \Phi$ is always nonpositive [the magnetosphere is never in a higher potential than the ionosphere (Lemaire and Scherer, 1983)]. Here $K$ is the Lyons-Evans-Lundin parameter, which in the present simulation is computed from

$K=\frac{\chi n e^{2}}{\sqrt{2 \pi m_{e} k_{B} T_{e}}}$

according to Fridman and Lemaire (1980, Eq. 12). The factor $\chi$ is the loss cone filling rate, which is a dimensionless number between 0 and 1 (for example, Janhunen, 1996).

Notice that although Fridman and Lemaire (1980) state that their Eq. (12) is valid only for potential drops that are neither too large nor too small (where "not too small" means much larger than the source plasma electron thermal energy), the equation is in fact valid even for arbitrarily small values, and it is a good numerical approximation to the full nonlinear Knight formula (Knight, 1973) in almost all practical cases (Janhunen and Olsson, 1998). Notice also that in the simulation we need the "inverted" current-voltage relationship, i.e., the voltage as a function of the current, for this reason it would be impractical to use the full nonlinear Knight formula.

\section{The electromagnetic ionosphere}

In the electrostatic ionosphere the magnetosphere gives the FAC and the ionosphere gives the potential and thus the convection velocity. An elliptic equation needs to be solved at the ionospheric plane. We now study what happens if the cause and effect are swapped.

We again start the interaction cycle from the $A B$, but instead of computing the FAC from its MHD definition we record the perpendicular component of the plasma velocity (the convection velocity). Then we compute the corresponding electric field $\mathbf{E}=\mathbf{B} \times \mathbf{v}$ at the $\mathrm{AB}$.

[Actually, we usually include the Hall term in MHD, thus in principle this equation should $\operatorname{read} \mathbf{E}=\mathbf{B} \times \mathbf{v}_{e}$ where $\mathbf{v}_{e}=\mathbf{v}-\mathbf{j} /($ en $)$ is the electron velocity, but the MHD Hall term can be important only in thin current sheets, and in the vicinity of the AB it is negligible. The electron pressure term is mathematically speaking of the same order as the Hall term, but we neglect it because in practice the electron pressure is smaller than the ion pressure. The parallel component of the electron pressure would be important in setting up the polar wind (Schunk, 1988), so modeling of the polar wind is outside the scope of the present simulation.]

The electric field then needs to be mapped through the force-free region to the ionospheric plane, including also the parallel potential drop. In principle this is a difficult problem, since the mapping of a nonelectrostatic electric field is fundamentally ambiguous. The mapping is unambiguous only in special cases, e.g. in case of electrostatic field, in which case the mapping of the vector can be reduced to mapping of a scalar potential. However, it may not be too bad an approximation for the simulation as a whole to use the electrostatic mapping even for a nonelectrostatic field, for the following reason. Errors in mapping affect only the trip from the $\mathrm{AB}$ surface to the ionosphere. The relevant magnetospheric phenomena interacting with the ionosphere do not take place in the vicinity of the AB surface (if they do, the AB surface has been placed at too high an altitude). "Mapping" outside the AB surface is performed correctly anyway, using the full set of MHD equations. Thus one expects that the errors introduced by the use of electrostatic mapping do not lead to a complete omission of some electromagnetic effects in the simulation as a whole, but, at worst, to a slight or moderate underestimation of the magnitude of these effects.

We now sketch briefly how to do the electrostatic mapping for a general electric field. The electric field $\mathbf{E}$ at $\mathrm{AB}$ surface at point $\mathbf{r}_{\mathrm{O}}$ is used to define a local potential, $\Phi(\mathbf{r})=\Phi_{\mathrm{o}}-\mathbf{E} \cdot\left(\mathbf{r}-\mathbf{r}_{\mathrm{o}}\right)$, taking only the first 
term of the Taylor expansion. After linearizing also the mapping function around $\mathbf{r}_{\mathrm{o}}$ we get the potential at the ionospheric plane as a linear function of the ionospheric coordinates (the function is valid only in the vicinity of the footpoint of $\mathbf{r}_{\mathrm{o}}$ ), which enables us to get the corresponding ionospheric electric field vector unambiguously. The parallel potential drop, if any, should be added to the potential at the ionosphere, in the same way as in the electrostatic approach.

Once the electric field (which is now not necessarily electrostatic, hence the name "electromagnetic ionosphere") has been obtained at the ionospheric plane, it is a straightforward operation to compute the corresponding horizontal current $\mathbf{J}=\Sigma \cdot\left(\mathbf{E}+\mathbf{v}_{n} \times \mathbf{B}\right)$. The divergence of the horizontal current gives the ionospheric FAC. The FAC can be mapped up to the magnetosphere easily because it is a scalar (recall, however, that we have to multiply with the magnetic field ratio to account for the changing flux tube cross section). The remaining problem is how to input the FAC on the AB to the MHD equations, i.e., how to turn the information of the FAC into knowledge of the variation field $\mathbf{B}_{1}$ components. We will study this next.

To illustrate the problem, assume first that the $A B$ is a plane and that the background magnetic field $\mathbf{B}_{0}$ is perpendicular to it. Choose the coordinate system such that the $\mathrm{AB}$ plane is the $X Y$ plane and $\mathbf{B}_{0}$ has only the $z$ component. In the vicinity of the $\mathrm{AB}$ the system is still approximately force-free (otherwise the AB has been placed at too high an altitude), thus we have

$\mathbf{B}_{0}=B_{0} \hat{z}, \quad \mathbf{B}_{1} \cdot \hat{\mathbf{z}}=0, \quad \partial_{z} \mathbf{B}_{1}=0, \quad \nabla \cdot \mathbf{B}_{1}=0$.

The FAC is only a function of $x$ and $y$. Using Ampère's law we obtain:

$j_{\|}(x, y)=\frac{1}{\mu_{0}}\left(\partial_{x} B_{1 y}-\partial_{y} B_{1 x}\right)$.

Representing the variation magnetic field $\mathbf{B}_{1}$ as the curl of a vector potential $A(x, y)$, we have $B_{1 x}=\partial_{y} A$, $B_{1 y}=-\partial_{x} A$. We obtain

$-\left(\partial_{x}^{2}+\partial_{y}^{2}\right) A(x, y)=\mu_{0} j_{\|}(x, y)$,

i.e., Poisson's equation at the $\mathrm{AB}$ surface for $A(x, y)$. For this construction to be meaningful we assumed (1) a planar AB surface, (2) constant $\mathbf{B}_{0}$, (3) $\mathbf{B}_{0}$ perpendicular to $\mathrm{AB}$, (4) force-free region in the vicinity of $\mathrm{AB}$, (5) small variation field, $\left|\mathbf{B}_{1}\right| \ll\left|\mathbf{B}_{0}\right|$.

A more realistic case would be a radial FAC distribution and spherical $A B$ surface. In this case the only approximation involved is the assumption that the FAC flows radially, while in reality it flows more or less along dipolar field lines. Using spherical coordinates $(r, \theta, \varphi)$, the FAC in this case is given by

$\mathbf{j}_{\|}(r, \theta, \varphi)=\frac{f(\theta, \varphi)}{r^{2}} \hat{\mathbf{r}}$

in order to have $\nabla \cdot \mathbf{j}_{\|}=0$. The vector potential $\mathbf{A}(r, \theta, \varphi)$ is always given by the explicit integral
$\mathbf{A}(r, \theta, \varphi)=\frac{\mu_{\mathrm{o}}}{4 \pi} \int d^{3} \mathbf{r}^{\prime} \frac{\mathbf{j}_{\|}\left(\mathbf{r}^{\prime}\right)}{\left|\mathbf{r}-\mathbf{r}^{\prime}\right|}$

(Jackson, 1975), from which it can be seen that the vector potential is independent of the radial coordinate $r$, that is, $\mathbf{A}(\alpha \mathbf{x})=\mathbf{A}(\mathbf{x})$ for any $\alpha>0$, which is also evident from the scale invariance of the current distribution. The differential equation for the vector potential, $-\nabla^{2} \mathbf{A}=\mu_{\mathrm{o}} \mathbf{j}_{\text {J }}$ therefore becomes a two-dimensional equation over the spherical $\mathrm{AB}$ surface, the three components of which are given by

$$
\begin{aligned}
& -2 A_{r}-2 \frac{\partial A_{\theta}}{\partial \theta}+\frac{\partial^{2} A_{r}}{\partial \theta^{2}}+\cot \theta\left(-2 A_{\theta}+\frac{\partial A_{r}}{\partial \theta}\right) \\
& -\frac{2}{\sin \theta} \frac{\partial A_{\varphi}}{\partial \varphi}+\frac{1}{\sin ^{2} \theta} \frac{\partial^{2} A_{r}}{\partial \varphi^{2}}=-\mu_{\mathrm{o}} f(\theta, \varphi), \\
& \quad \frac{1}{\sin ^{2} \theta}\left(-A_{\theta}-2 \cos \theta \frac{\partial A_{\varphi}}{\partial \varphi}+\frac{\partial^{2} A_{\theta}}{\partial \varphi^{2}}\right) \\
& +2 \frac{\partial A_{r}}{\partial \theta}+\cot \theta \frac{\partial A_{\theta}}{\partial \theta}+\frac{\partial^{2} A_{\theta}}{\partial \theta^{2}}=0, \\
& -\frac{A_{\varphi}}{\sin ^{2} \theta}+\frac{1}{\sin \theta}\left(2 \frac{\partial A_{r}}{\partial \varphi}+2 \cot \theta \frac{\partial A_{\theta}}{\partial \varphi}+\frac{1}{\sin \theta} \frac{\partial^{2} A_{\varphi}}{\partial \varphi^{2}}\right) \\
& +\cot \theta \frac{\partial A_{\varphi}}{\partial \theta}+\frac{\partial^{2} A_{\varphi}}{\partial \theta^{2}}=0 .
\end{aligned}
$$

This is much more complicated than Poisson's equation in the planar case, but it is still two-dimensional.

The most realistic assumption would be an FAC distribution following dipolar field lines and the shape of the AB surface again being spherical. The problem is again to compute the magnetic field on the AB surface caused by the FAC distribution. Now we can see that the problem setup is somewhat ambiguous, because one has to assume some closure for the FAC current pattern. For example, assuming that the FAC follows dipolar field lines up to the equatorial plane would create a nonphysical discontinuity in the current on the equatorial plane, as the FAC patterns from the southern and northern hemispheres would probably not match. However, it is probable that in most cases the way this ambiguity is solved has only a small effect on the computed magnetic perturbation on the AB surface, because the image of the main FAC systems on the AB surface is rather far from the equatorial plane. Thus, a possible brute-force method to compute the magnetic perturbation would be to extend the FAC pattern threading the $\mathrm{AB}$ surface along dipolar field lines and solving the $3 \mathrm{D}$ Poisson equation $-\nabla^{2} \mathbf{A}=\mu_{\mathrm{o}} \mathbf{j}_{\|}$in the near-Earth region. In order to prevent boundaries from affecting the solution at the AB surface, the grid used to solve this equation should include regions from both sides of the AB surface, thus it must be a different grid to that used in the MHD equations.

A remaining problem is how to keep the variation field $\mathbf{B}_{1}$ divergence-free in the vicinity of the $\mathrm{AB}$ surface. Ways to deal with this issue depend on how the magnetic field is kept divergence-free elsewhere in the 
MHD box. Our current GUMICS-3 simulation solves a 3D Poisson equation at every $8 \mathrm{~s}$ as a divergencecleaning procedure. Other strategies include the eightwave Riemann solver of Powell (Powell et al., 1995), which allows for nonzero divergence in the MHD equations but includes terms which should keep its magnitude small, and the manifestly divergence-free method of Dai and Woodward (1996), in which the magnetic field is stored as cell surface averages.

\section{Discussion and conclusions}

From the physical point of view, the ionosphere and the magnetosphere should be considered as interacting systems, which maintain a self-consistent electric field and current distribution. Thus one may think that the magnetosphere gives the field-aligned current pattern, to which the ionosphere responds by giving an electric field pattern to the magnetosphere (the electrostatic approach), or one may think that the magnetosphere gives the electric field pattern, to which the ionosphere responds by giving the FAC pattern (the electromagnetic approach). In the former case the ionosphere drives the magnetospheric (and ionospheric) convection, whereas in the latter case the ionosphere acts as a source of FAC. Both approaches are possible, but for realization in an MHD simulation, only one of the approaches can be chosen. Thus far only the electrostatic approach has been used in actual simulations. It would be advantageous if also the electromagnetic approach could be implemented so that the results could be compared. That might provide us with additional physical insight.

We investigated the possibility of an electromagnetic approach. We derived formulas for inputting the FAC into an approximately force-free MHD region. In the electromagnetic approach we need not solve an elliptic equation at the ionospheric plane, but instead we must solve a Poisson-type equation (which would be exactly Poisson's equation if magnetic field were radial) at the AB surface. The computational work associated with the Poisson-type equation is likely to be somewhat smaller than the work needed to solve the more general elliptic equation at the ionospheric plane, which would mean that the electromagnetic approach could be faster.

Two problem areas remain, which we did not fully resolve in this paper: the problem of mapping the electric field (vector quantity) from the $\mathrm{AB}$ surface through the force-free region into the ionospheric plane, and the problem of keeping the $\mathbf{B}_{1}$ field divergence-free in the vicinity of the $\mathrm{AB}$ surface. The mapping of the electric field is unambiguous only in the case of electrostatic field. As a first trial, the electrostatic mapping could be used, in which case the scheme that we named "electromagnetic" is actually not fully electromagnetic. However, if the AB surface is not too far from the ionosphere, as should be the case anyway, the error made here is likely to be small. The second problem is how to keep the magnetic field divergencefree near the AB surface. This is closely related to the divergence cleaning strategy used elsewhere in the MHD simulation box and thus it is outside the scope of this paper. We expect the second problem not to be too difficult.

If these problems are reasonably solved, then an attempt should be made to implement the electromagnetic approach for comparison purposes. It is too early to predict now which one of the approaches will turn out to be preferrable, but in any case the electromagnetic approach should be investigated further.

Acknowledgements. The author thanks Kirsti Kauristie, Anssi Mälkki, Olaf Amm and Hannu Koskinen for useful discussions on various, more or less related topics.

Topical Editor K.-H. Glapmeier thanks J. Raeder and M. L. Goodman for their help in evaluating this paper.

\section{References}

Amm, O., Comment on "A three-dimensional iterative mapping procedure for the implementation of an ionosphere-magnetosphere anisotropic Ohm's law boundary condition in global magnetohydrodynamic simulations" by Michael L. Goodman, Ann. Geophysicae, 14, 773-774, 1996.

Dai, W., and P. R. Woodward, On the divergence-free condition and conservation laws in numerical simulations for supersonic magnetohydrodynamical flows, Minnesota Supercomputer Institute Research Report, UMSI 96/205, 1996.

Fedder, J. A., J. G. Lyon, S. P. Slinker and C. M. Mobarry, Topological structure of the magnetotail as a function of interplanetary magnetic field direction, J. Geophys. Res., 100, 3613-3621, 1995.

Fridman, M., and J. Lemaire, Relationship between auroral electrons (sic) fluxes and field aligned electric potential difference, J. Geophys. Res., 85, 664-670, 1980.

Goodman, M. L., A three-dimensional, iterative mapping procedure for the implementation of an ionosphere-magnetosphere anisotropic Ohm's law boundary condition in global magnetohydrodynamic simulations, Ann. Geophysicae, 13, 843-853, 1995.

Goodman, M. L., Reply to Olaf Amm's comment on "A threedimensional iterative mapping procedure for the implementation of an ionosphere-magnetosphere anisotropic Ohm's law boundary condition in global magnetohydrodynamic simulations" by Michael L. Goodman, Ann. Geophysicae, 14, 775-775, 1996.

Jackson, J. D., Classical electrodynamics, Wiley, New York, 1975.

Janhunen, P., GUMICS-3 - a global ionosphere-magnetosphere coupling simulation with high ionospheric resolution, in Proc. environmental modelling for space-based applications, ESA SP392 18-20 Sept. 1996, ESTEC, The Netherlands, pp. 233-239, 1996.

Janhunen, P., and H. E. J. Koskinen, The closure of Region-1 fieldaligned current in MHD simulation, Geophys. Res. Lett., 24, 1419-1422, 1997.

Janhunen, P. and A. Olsson, The current-voltage relationship revisited: exact and approximate formulas with almoste general validity for hot magnetospheric electrons for bi-Maxwellian and kappa distributions, Ann. Geophysicae, in press, 1998.

Janhunen, P., H. E. J. Koskinen and T. I. Pulkkinen, A new global ionosphere-magnetosphere coupling simulation utilizing locally varying time step, in Proc. 3rd Int. Conf. Substorms (ICS3), ESA SP-389 Versailles, France, May 12-17, ESA SP, pp. 205-210, 1996.

Knight, S., Parallel electric fields, Planet. Space Sci., 21, 741-750, 1973. 
Lemaire, J. and M. Scherer, Field-aligned current density versus electric potential characteristics for magnetospheric flux tubes, Ann. Geophysicae, 1, 91-96, 1983.

Oppenheim, M., Nonlinear simulations and theory of the FarleyBuneman instability in the E-region ionosphere, Ph.D. thesis, Cornell University, 1995.

Powell, K. G., P. L. Roe, R. S. Myong, T. Gombosi and D. DeZeeuw, An upwind scheme for magnetohydrodynamics, AIAA Paper, 95-1704-CP, 1995.

Press, W. H., S. A. Teukolsky, W. T. Vetterling, and B. P. Flannery, Numerical recipes in C: the art of scientific computing, 2nd edn, Cambridge University Press, cambridge, 1992.
Raeder, J., R. J. Walker and M. Ashour-Abdalla, The structure of the distant geomagnetic tail during long periods of northward IMF, Geophys. Res. Lett., 22, 349-352, 1995.

Schunk, R. W., The polar wind, in Modeling magnetospheric plasma, Geophys. Monogr. 44, eds. T. E. Moore and J. H. Waite Jr., AGU, Washington, D.C., pp 219-228 1988.

Tanaka, T., Generation mechanisms for magnetosphere-ionosphere current systems deduced from a three-dimensional MHD simulation of the solar wind-magnetosphere-ionosphere coupling processes, J. Geophys. Res., 100, 12057-12074, 1995. 